\title{
Aim-P: Um arcabouço de apoio a implantação de práticas de melhorias de pessoas em organizações de software
}

\author{
Leandro M. de Oliveira, Maxwell S. dos Santos, Marcelo A. S. Turine, Débora M. \\ B. Paiva, Geraldo B. Landre, Márcio A. I. da Silva, Maria Istela Cagnin
}

Faculdade de Computação - Universidade Federal de Mato Grosso do Sul (UFMS) Caixa Postal 549 - 79070-900 - Campo Grande - MS - Brazil

\{leandro.oliveira, maxwell.santos\}@ufms.br, \{turine, debora, geraldo, marcio, istela\} @facom.ufms.br

\begin{abstract}
Companies are facing a novel economic paradigm which establishes new needs. These needs forces companies to update the way their employees are managed. In this context, P-CMM (People Capability Maturity Model) is capable to drive companies on people management and development. This paper presents Aim-P, a framework that aims to support gradual and incremental deployment of P-CMM in micro, small and medium companies, providing mechanism and guidelines for this. Additionally, in order to observe the use of Aim-P, a case study in a real environment is being conducted.
\end{abstract}

Resumo. As organizações estão participando de um novo paradigma econômico que estabelece novas necessidades. Esse novo paradigma vem pressionando as organizações em direção à modernização na forma de gerir pessoas. Nesse contexto foi proposto o P-CMM (People Capability Maturity Model), um modelo capaz de guiar as organizações em direção à gestão e ao desenvolvimento de pessoas. Este artigo apresenta o arcabouço Aim-P que visa apoiar a implantação do P-CMM de maneira gradual e incremental em micro, pequenas e médias organizações de software; fornecendo mecanismos e diretrizes para isso. Para observar o uso do arcabouço proposto está sendo conduzido um estudo de caso em uma organização real.

\section{Introdução}

Desde o surgimento da Engenharia de Software ocorreram mudanças significativas no mercado de software, trazendo novos desafios e buscas por novas soluções e abordagens. As organizações estão participando de um novo paradigma econômico que estabelece a necessidade da inovação contínua, da geração de produtos e serviços com maior valor agregado, e da preocupação com a satisfação do cliente (BECKER et al., 2001). Esse cenário vem afetando a coordenação do trabalho, bem como as relações entre as organizações e seus profissionais.

Cada vez mais as organizações necessitam do conhecimento e do envolvimento de seus profissionais bem como da articulação desses em equipes, de forma a alcançar e manter sua competitividade. $\mathrm{O}$ diferencial dessas organizações não ocorre apenas pela posse de tecnologias. Sua verdadeira fonte de vantagem competitiva é aquela obtida por meio das pessoas. Além disso, as pessoas vêm adquirindo consciência da necessidade de 
gerir sua carreira e passam a requerer das organizações condições mais objetivas de aperfeiçoamento profissional (DE GEUS, 2002).

Observando esse contexto, um importante modelo para gestão de pessoas foi proposto pelo Software Engineering Institute (SEI), denominado People Capability Maturity Model - P-CMM (CURTIS et al., 2009). Esse modelo auxilia as organizações na implementação de um conjunto de práticas de gestão e de desenvolvimento de pessoas, visando que essa aja como um centro independente de inteligência sem, contudo, desviar-se da estratégia e dos objetivos organizacionais (CURTIS et al., 2009).

Como ocorre com modelos de maturidade e capacidade no contexto de software (por exemplo, CMMI (SEI, 2010), MR-MPS (SOFTEX, 2012) e ISO/IEC 15504 (ISO, 2004)), o P-CMM provê apenas "o que" deve ser feito para desenvolver as pessoas que fazem parte de uma organização, cujas práticas melhoram continuamente a capacidade de sua força de trabalho, e não "como" tais práticas podem ser implantadas. Adicionalmente, observou-se que o SEI não fornece um mecanismo para analisar a maturidade das organizações no contexto de sua força de trabalho; como é o caso do SCAMPI (SEI, 2010) e do MA-MPS (SOFTEX, 2013) no contexto de processos de software.

Diante disso, foi realizado um levantamento na literatura para identificar mecanismos de apoio à implantação do P-CMM, bem como modelos ou métodos de avaliação de maturidade em conformidade com esse modelo. Entretanto, pelas buscas realizadas não foram encontrados na literatura trabalhos com esses enfoques.

Buscando preencher a lacuna encontrada, este artigo apresenta um arcabouço ${ }^{1}$ denominado Aim-P (Arcabouço para a Implantação de Melhoria de Pessoas) que busca apoiar a implantação do P-CMM de maneira gradual e incremental em micro, pequenas e médias organizações de software. Adicionalmente, o Aim-P oferece diretrizes para que as organizações realizem uma auto-avaliação em conformidade com o P-CMM.

Para observar a aplicabilidade do Aim-P, um estudo de caso está sendo conduzido no Laboratório de Engenharia de Software (Ledes) da Facom/UFMS. Resultados preliminares mostram que o uso do Aim-P é relevante para auxiliar na implantação do modelo de melhoria de pessoas P-CMM, apoiando a implantação de práticas esperadas pelos objetivos do modelo.

A escrita deste artigo está organizada da seguinte forma: $\mathrm{Na}$ Seção 2 é apresentada uma visão geral do modelo P-CMM; na Seção 3 são apresentados os trabalhos relacionados; na Seção 4 é apresentado o arcabouço Aim-P; na Seção 5 é apresentado o estudo de caso que está sendo conduzido; e na Seção 6 são apresentadas as conclusões e sugestões de trabalhos futuros.

\section{P-CMM}

O P-CMM é um modelo de maturidade que auxilia as organizações na implementação de um conjunto de práticas de gestão e de desenvolvimento de sua força de trabalho (CURTIS et al., 2009).

\footnotetext{
${ }^{1}$ Conjunto de elementos (atividades, artefatos, etc) usados para resolver um problema ou alcançar um determinado objetivo.
} 
Esse modelo está estruturado em cinco níveis de maturidade, conforme ilustrados na Figura 1, em que a organização transforma sua cultura a cada passagem de nível e, dessa maneira, possibilita o estabelecimento e a evolução das práticas de atração, aprimoramento, motivação e retenção de pessoas (CURTIS et al., 2009). O PCMM possibilita o desenvolvimento de um ambiente propício aos indivíduos experimentarem oportunidades de crescimento de carreira e motivação, facilitando seu engajamento aos objetivos organizacionais.

Cada nível representa o alicerce necessário para o nível imediatamente acima. À medida que a organização amadurece a cada mudança de nível do P-CMM, suas práticas de gestão de pessoas apontam em direção aos objetivos estratégicos e impactam cada vez mais o nível de capacidade da força de trabalho.

A estrutura em níveis do P-CMM é derivada do CMM (Capability Maturity Model), que é o modelo de maturidade de processos também empregado no Capability Maturity Model Integration for Development (CMMI-DEV) (SEI, 2010). A versão do P-CMM utilizada por este trabalho é a mais recente e já está em conformidade com o CMMI.

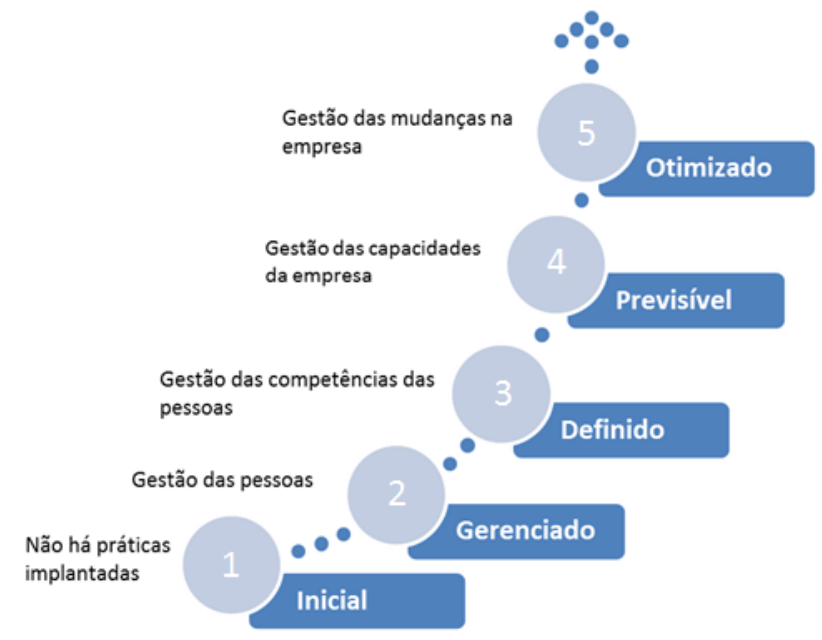

Figura 1 - Estrutura geral do P-CMM (adaptado de Curtis et al. (2009))

Cada nível de maturidade, com exceção do nível 1, é composto por um conjunto de áreas de processo, cada qual representando um importante processo organizacional de gestão de pessoas. Cada área de processo é constituída por objetivos que representam os requisitos que uma organização deve satisfazer, independentemente de seu tamanho, localização ou produto, de forma a estabelecer a habilidade da área de processo em atuar sobre a capacidade da força de trabalho. Um nível de maturidade somente é alcançado quando todos os objetivos de cada área de processo que o compõe forem satisfeitos, descrevendo de forma precisa o comportamento da organização.

Para atender os objetivos das áreas de processo existe um desmembramento entre as práticas: práticas de implementação e práticas de institucionalização. As práticas de implementação referem-se às práticas e procedimentos implementados pela organização para satisfazer os objetivos de uma área de processo, enquanto as práticas de institucionalização referem-se aos procedimentos implementados para assegurar que a organização mantenha o compromisso quanto ao desenvolvimento de pessoas e a capacidade para desempenhar as práticas e atividades de trabalho da organização. 
As práticas de implementação foram propostas para estabelecer melhorias em grupos ou indivíduos, ou mesmo para executar avaliações dos procedimentos, enquanto as práticas de institucionalização visam dificultar o retrocesso das práticas de implementação a estados anteriores de maturidade.

No Quadro 1 são apresentados os objetivos gerais de cada nível, além das áreas de processos contempladas por cada um dos níveis.

Quadro 1 - Áreas de processo e objetivos gerais de cada nível do P-CMM (adaptado de Curtis et al. (2009))

\begin{tabular}{|c|c|c|c|}
\hline Nível & Foco & Objetivos & Áreas de Processo \\
\hline $\begin{array}{l}\text { Nível 1 } \\
\text { Inicial }\end{array}$ & Não há & Não há & Não há \\
\hline \multirow{6}{*}{$\begin{array}{l}\text { Nível } 2 \\
\text { Gerenciado }\end{array}$} & \multirow{6}{*}{$\begin{array}{l}\text { Gestão de } \\
\text { pessoas }\end{array}$} & \multirow{6}{*}{$\begin{array}{l}\text { Gestão e desenvolvimento } \\
\text { da equipe pelos gerentes }\end{array}$} & Alocação de pessoal (Staffing) \\
\hline & & & Comunicação e coordenação \\
\hline & & & Ambiente de trabalho \\
\hline & & & Gestão de desempenho \\
\hline & & & Treinamento e desenvolvimento \\
\hline & & & Compensação \\
\hline \multirow{7}{*}{$\begin{array}{l}\text { Nível } 3 \\
\text { Definido }\end{array}$} & \multirow{7}{*}{$\begin{array}{l}\text { Gestão das } \\
\text { competências } \\
\text { das pessoas }\end{array}$} & \multirow{7}{*}{$\begin{array}{l}\text { Desenvolvimento de } \\
\text { competências e grupos de } \\
\text { trabalho, alinhando-os aos } \\
\text { objetivos e estratégias do } \\
\text { negócio }\end{array}$} & Análise de competências \\
\hline & & & Planejamento dos recursos humanos \\
\hline & & & Desenvolvimento de competências \\
\hline & & & $\begin{array}{l}\text { Desenvolvimento de práticas baseadas em } \\
\text { competências }\end{array}$ \\
\hline & & & Desenvolvimento de carreiras \\
\hline & & & Desenvolvimento de grupos de trabalho \\
\hline & & & Cultura participativa \\
\hline \multirow{6}{*}{$\begin{array}{l}\text { Nível } 4 \\
\text { Previsível }\end{array}$} & \multirow{6}{*}{$\begin{array}{l}\text { Gestão das } \\
\text { capacidades } \\
\text { da empresa }\end{array}$} & \multirow{6}{*}{$\begin{array}{l}\text { Integração das competências } \\
\text { no trabalho e gerenciamento } \\
\text { quantitativo do desempenho }\end{array}$} & Integração de competências \\
\hline & & & Grupos de trabalho autônomos \\
\hline & & & Ativos baseados em competências \\
\hline & & & Gestão quantitativa do desempenho \\
\hline & & & Gestão da capacidade organizacional \\
\hline & & & Orientação e aconselhamento \\
\hline \multirow{3}{*}{$\begin{array}{l}\text { Nível } 5 \\
\text { Otimizado }\end{array}$} & \multirow{3}{*}{$\begin{array}{l}\text { Gestão das } \\
\text { mudanças na } \\
\text { empresa }\end{array}$} & \multirow{3}{*}{$\begin{array}{l}\text { Instauração da melhoria } \\
\text { contínua dos processos e } \\
\text { alinhamento do grupo de } \\
\text { trabalho com a capacidade } \\
\text { organizacional }\end{array}$} & Melhoria contínua da capacidade \\
\hline & & & $\begin{array}{lll}\begin{array}{l}\text { Alinhamento } \\
\text { organizacional }\end{array} & \text { do } & \text { desempenho } \\
\end{array}$ \\
\hline & & & Inovação contínua de recursos humanos \\
\hline
\end{tabular}

\section{Trabalhos Relacionados}

Mecanismos de apoio à implantação de modelos de melhorias foram encontrados na literatura. Porém, todos pertencem ao contexto de implantação de melhoria em processos de software como é o caso do modelo IDEAL (MCFEELEY, 1996), da estratégia SPI-KM (SANTOS et al., 2007) e do IAMPS (BENITES, 2012).

O IDEAL é um modelo iterativo concebido para apoiar a melhoria de processo de desenvolvimento de software. Seu desenvolvimento apoia-se em experiências de melhoria em grandes empresas e instituições governamentais norte-americanas. A estratégia SPI-KM é baseada no modelo de referência CMMI e em normas internacionais (ISO/IEC 12207 (2008) e ISO/IEC 15504 (2004)) para a implantação de melhoria de processo baseada em conhecimento. O IAMPS é um processo incremental e 
iterativo de implantação de melhoria de processo baseado no modelo de maturidade brasileiro MPS.BR (SOFTEX, 2012), em conjunto com métodos ágeis. Oferece suporte para que pequenas e médias empresas possam melhorar seus processos ágeis e é baseado no modelo IDEAL e na estratégia SPI-KM.

Com as buscas realizadas, não foram encontrados trabalhos diretamente relacionados a este artigo. Todavia, foram encontrados trabalhos sobre a utilização de modelos de gestão de pessoas com melhoria de processo de software, relatos de experiência na implantação de níveis de maturidade do modelo P-CMM e experiências na criação e utilização de ferramentas para alcançar determinados objetivos esperados pelo modelo P-CMM (GALVÃO e MAGALHÃES, 2005; CHEN et al., 2012; HEFLEY, 1995).

\section{Arcabouço Aim-P}

O Aim-P visa auxiliar as micros, pequenas e médias empresas brasileiras, que desenvolvem software, a implantar um modelo de melhoria de pessoas, mais especificamente o P-CMM, bem como a avaliar a maturidade das mesmas quanto a esse modelo.A estrutura geral do arcabouço Aim- $\mathrm{P}^{2}$ é baseada na estrutura de implantação de melhorias do modelo IDEAL e do processo IAMPS, bem como nos modelos de ciclo de vida de desenvolvimento de software mais tradicionais, como o modelo incremental e espiral (SOMMERVILLE, 2010). Justifica-se o uso desses mecanismos uma vez que as características das empresas brasileiras de software foram levadas em consideração em sua concepção, isto é, o arcabouço foi desenvolvido pensando em sua aplicabilidade em micros, pequenas e médias empresas que, na maioria das vezes, possuem recursos financeiros e humanos limitados. Assim, a melhoria de pessoas pode ser feita de maneira gradual e de acordo com as necessidades, interesses e recursos disponíveis da organização.

$\mathrm{O}$ arcabouço é dividido em cinco estágios, conforme ilustrados na Figura 2. O primeiro estágio do Aim-P, "Estabelecer equipes", é responsável por oferecer um guia para a formação de equipes (inclusive equipes ágeis, caso seja de interesse da organização); o estágio "Avaliar pessoas", é responsável por avaliar a equipe quanto a aderência em relação às características de equipes ágeis (se for de interesse da organização) e também uma auto-avaliação para mensurar quais objetivos do modelo PCMM estão sendo atendidos pelas pessoas que compõem as equipes de desenvolvimento de software da organização; o estágio "Definir objetivos de implantação de melhorias" é responsável por definir quais são os objetivos do projeto da organização de implantação de melhoria de pessoas na organização; o estágio "Planejar iteração de implantação de melhorias" é responsável pelo planejamento de cada etapa de melhoria; por fim, no estágio "Executar implantação de melhorias" é feita a execução do que foi planejado no estágio anterior.

\footnotetext{
${ }^{2}$ Descrição detalhada do arcabouço (atividades de cada estágio, entradas, saídas, pré-condições, artefatos, responsáveis, etc.) pode ser encontrada em Oliveira (2013).
} 


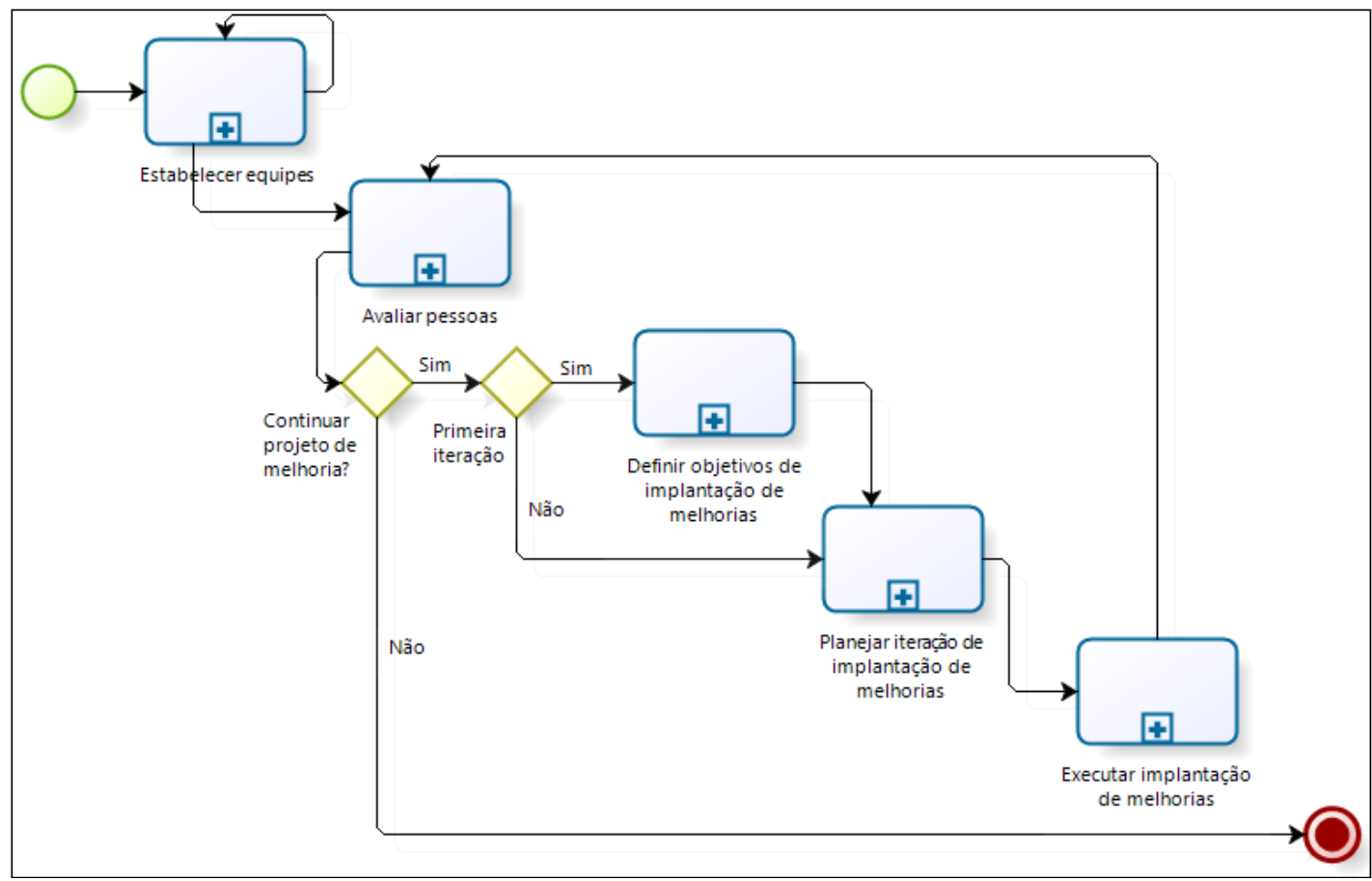

Figura 2 - Visão geral do arcabouço Aim-P

Nas subseções a seguir é apresentado um detalhamento de cada um dos cinco estágios supracitados. Devido a restrição de espaço, apenas os artefatos mais importantes produzidos em cada estágio são discutidos.

\subsection{Estágio: Estabelecer equipes}

Durante a participação do primeiro autor deste trabalho em um projeto de implantação de melhoria de processos de software (BENITES et al., 2012) foi possível perceber que, apesar de parecer algo trivial, a formação de equipes é uma tarefa que gera uma quantidade razoável de reclamações e insatisfações por parte dos colaboradores.

Visando solucionar os problemas observados, este estágio consiste em formar a equipe tomando ou não como base diretrizes fornecidas pelo arcabouço. A concepção dessas diretrizes foram baseadas, em geral, nas práticas e valores do Manifesto Ágil (AGILE ALLIANCE, 2001) relacionados a equipes. A definição de equipe adotada por este trabalho é a mesma do TSP (Team Software Process), ou seja, uma equipe consiste de pelo menos três pessoas que compartilham os mesmos objetivos, possuem papéis estabelecidos e dependem uma das outras para atingirem seus objetivos (HUMPHREY, 2000).

As diretrizes providas pelo Aim-P oferecem um guia para formar uma equipe que mantém as características de métodos ágeis, e também possibilita selecionar indivíduos de forma que todas as habilidades necessárias sejam contempladas, isto é, exista pelo menos uma pessoa da equipe que seja capaz de realizar uma tarefa necessária para o bom andamento do projeto de desenvolvimento de software.

A seguir é apresentado um exemplo de uma das diretrizes propostas e que pode ser utilizada para a formação de equipes. As demais diretrizes estão disponíveis em Oliveira (2013). 


\section{Escolhendo os membros da equipe}

"A escolha dos membros de uma equipe deve ser feita pelo setor de recursos humanos ou pelo responsável em recursos humanos da organização. Por padrão, as equipes formadas serão pequenas (de 3 a 8 membros) então se espera que as equipes sejam multidisciplinares e que os colaboradores sejam capazes de assumir mais de um papel, caso necessário. A definição dos critérios para a escolha dos membros da equipe deve levar em consideração tais necessidades."

Além disso, baseado no modelo de evolução de grupos de trabalho, proposto por Tuckman (2001), o arcabouço Aim-P descreve neste estágio como ocorre a evolução das equipes formadas e quais são as etapas de evolução dessas equipes a fim de que a equipe cresça mais forte, enfrente desafios, lide com os problemas, encontre soluções e, eventualmente, produza os resultados desejados.

\subsection{Estágio: Avaliar Pessoas}

Este estágio diz respeito a análise do estado das pessoas, que é executada tanto no início do projeto de implantação de melhorias de pessoas quanto durante o mesmo para acompanhar a evolução das pessoas. No primeiro caso, obtêm-se um diagnóstico do estado atual das pessoas para apoiar no estabelecimento de objetivos de implantação de melhorias de pessoas (estágio "Definir objetivos de implantação de melhorias"). No segundo caso, observa-se a evolução das equipes e das pessoas após o término de cada iteração de implantação de melhorias.

Para a concepção deste estágio foi conduzida uma abstração dos objetivos de modelos de avaliação de maturidade de processos de software, como é o caso do MAMPS (SOFTEX, 2013), que é o método de avaliação do MPS.BR; e do SCAMPI (SCAMPI, 2011), que é o modelo de avaliação do CMMI (SEI, 2010).

A avaliação de pessoas pelo arcabouço Aim-P é conduzida de duas formas. Na primeira, a equipe é avaliada considerando como critérios de avaliação características de equipes comumente descritas em métodos ágeis. A partir dos resultados desse primeiro tipo de avaliação é possível averiguar a aderência da equipe quanto às características de equipes ágeis. Esse tipo de avaliação é opcional, pois nem todas as organizações utilizam métodos ágeis como modelo de processo de desenvolvimento de software.

No outro tipo de avaliação, as pessoas são avaliadas considerando como critérios de avaliação os objetivos de melhoria planejados para serem implantados na iteração corrente ou, em uma avaliação preliminar, para identificar o estado de aderência das pessoas da organização aos objetivos do P-CMM. Dessa forma, a partir dos resultados dessa segunda avaliação é possível identificar o grau de implantação dos objetivos de melhorias planejados: totalmente, parcialmente ou não implantado. Isso fornecerá subsídios para planejar a próxima iteração de implantação de melhoria, pois os objetivos de melhoria parcialmente implantados e não implantados retornarão para a "Lista de melhorias", que é um artefato produzido e atualizado no estágio "Definir objetivos de implantação de melhorias". Ressalta-se que para a avaliação de uma equipe é possível considerar apenas um dos tipos de avaliação ou ambos, isso será definido pela equipe de implantação e de acordo com o objetivo da avaliação.

Dentre os artefatos utilizados durante esse estágio destaca-se o "Modelo do formulário de avaliação" (Figura 3). Nesse modelo são descritas as práticas de cada área de processo de cada nível do P-CMM. Para utilizá-lo a equipe de implantação de 
melhorias deve configurá-lo para apoiar a avaliação da equipe de acordo com os critérios desejados. Após essa configuração a avaliação é realizada gerando o "Formulário de avaliação" (preenchido) e o "Relatório de execução da avaliação". Esse último apresenta informações consolidadas da avaliação conduzida e é apresentado aos gestores da organização para as tomadas de decisões e direcionar o projeto de implantação de melhorias em conformidade com os interesses e a disponibilidade de recursos da organização.

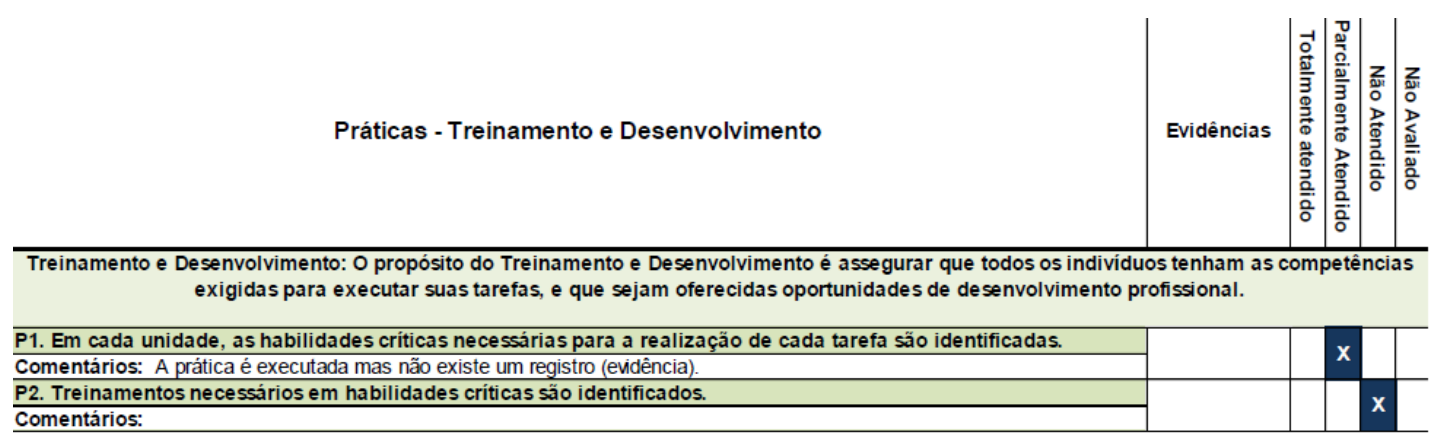

Figura 3 - Trecho do "Modelo de formulário de avaliação"

\subsection{Estágio: Definir objetivos de implantação de melhorias}

Uma vez passado pelo estabelecimento da equipe (estágio "Estabelecer equipes") e pela primeira avaliação da equipe de desenvolvimento, por meio do estágio "Avaliar Pessoas", o estágio para a definição de objetivos de implantação de melhorias do projeto de melhorias de pessoas é iniciado.

O resultado da avaliação da equipe de desenvolvimento permite identificar os pontos fracos da equipe e provê conhecimento para definir os objetivos de implantação de melhorias em áreas de processo do P-CMM onde existe maior necessidade de mudança ou permite desconsiderar áreas de processos já atendidas ou parcialmente atendidas pela equipe.

Assim, o intuito deste estágio é levantar as necessidades de melhorias tomando como base a opinião de todos os colaboradores e, em seguida, definir os reais objetivos da implantação de melhoria de pessoas e alinhá-los de forma que o projeto de melhorias da organização esteja baseado em um plano exequível, de acordo com os recursos disponíveis.

Dentre os principais artefatos utilizados nesse estágio destacam-se: o "Questionário para identificar necessidades de melhorias" (Figura 4), o "Resultado consolidado dos questionários" e o "Relatório estatístico das necessidades de melhorias" (Figura 5). O primeiro artefato citado é obtido a partir da configuração de um modelo oferecido pelo arcabouço, levando em consideração as várias esferas da organização (dos desenvolvedores aos gestores).

O "Questionário para identificar necessidades de melhorias" é organizado em três partes: a primeira parte é responsável pela caracterização do respondente, por meio de dados específicos sobre a posição que ocupa na organização, formação profissional, conhecimento a respeito de modelos de melhoria e informações pessoais; a segunda parte do questionário permite identificar as áreas de processo do(s) nível(is) de interesse do P-CMM de maior prioridade para serem implantadas na organização, de acordo com a opinião de cada respondente; e a terceira parte do questionário possibilita a 
identificação de quais objetivos de cada área de processo de cada nível de interesse possuem maior prioridade para a implantação de melhoria, também de acordo com a opinião de cada respondente.

Exemplificando a segunda parte do questionário para o nível 2 do P-CMM, foram definidos seis níveis de prioridade, um para cada área de processo desse nível. Os respondentes devem atribuir uma prioridade distinta para cada uma das áreas. $\mathrm{Na}$ terceira parte do questionário, para cada conjunto de objetivos de cada área, é necessário que o respondente atribua prioridade de um a dez para cada um dos objetivos, sem repetições de prioridades entre objetivos da mesma área de processo. Por exemplo, para os objetivos da área de processo Alocação de Colaboradores (Staffing), o respondente deve atribuir prioridades distintas entre um e dez para cada um dos objetivos, e assim sucessivamente para as outras cinco áreas de processo restantes.

O segundo artefato relevante deste estágio ("Resultado consolidado dos questionários") é obtido por meio da tabulação das informações extraídas das respostas do "Questionário para identificar necessidades de melhorias". Para gerá-lo é necessário inicialmente realizar a tabulação dos dados dos questionários respondidos pela equipe da organização. O terceiro artefato importante deste estágio ("Relatório estatístico das necessidades de melhorias") é um documento que consolida as informações obtidas no questionário e que é apresentado aos patrocinadores, servindo como fonte de informação para tomadas de decisões em relação aos pontos de melhorias que precisam e/ou serão atacados pelo projeto de melhoria. Esse relatório é obtido a partir da somatória das médias por perfil de respondente, multiplicado pelo peso de cada perfil somado à prioridade em relação a área de processo de determinado nível de interesse. $\mathrm{O}$ peso de cada perfil deve ser definido pela equipe de implantação e de acordo com a importância das posições dentro da organização.

\section{Áreas de Processo - P-CMM Nivel 2 \\ Priorize de 1 (menor prioridade) até 6 (maior prioridade) as áreas de processo de acordo com a sua importância. \\ Por favor não responda as questões determinando prioridades iguais, devido a limitações do formulário não foi possivel restringir estes casos.}

Staffing (Alocação de Colaboradores): 0 propósito do Staffing é estabelecer um processo pelo qual o comprometimento profissional é acompanhado e pessoas qualificadas são recrutadas, selecionadas e alocadas para suas tarefas.

Comunicação e Coordenação: $O$ propósito da Comunicação e Coordenação é estabelecer uma comunicação por toda a organização, garantindo que as pessoas compartilhem informação e coordenem suas atividades eficientemente.

Figura 4 - Trecho da segunda parte do questionário para identificar necessidades de melhorias (nível 2 do P-CMM) 


\begin{tabular}{|c|c|c|c|c|}
\hline \multicolumn{5}{|c|}{ PARTE III - Relatório estatístico das necessidades de melhoria } \\
\hline & \multicolumn{4}{|c|}{$\begin{array}{c}\text { CÁLCULO DA PRIORIDADE DOS OBJETIVOS } \\
\text { POR ÁREAS DE PROCESSO }\end{array}$} \\
\hline ÁREAS DE PROCESSO & Obj 1 & Obj 2 & Obj 3 & Obj 4 \\
\hline staffing & 418,56 & 345,33 & 464,33 & 422,67 \\
\hline comunicação & 292,33 & 333,94 & 378,00 & \\
\hline ambiente & 306,00 & 255,00 & & \\
\hline desempenho & 185,78 & 209,89 & 322,22 & 203,39 \\
\hline treinamento & 266,00 & 208,56 & & \\
\hline compensação & 111,00 & 114,67 & 98,33 & \\
\hline
\end{tabular}

Figura 5 - Trecho do relatório estatístico das necessidades de melhorias (nível 2 do P-CMM)

\subsection{Estágio: Planejar iteração de implantação de melhorias}

Neste estágio é feito o planejamento da implantação das melhorias definidas e priorizadas no estágio "Definir objetivos de implantação de melhorias". Ele é responsável por produzir e comunicar um plano adequado à execução de uma iteração de implantação. Dessa forma, dado um conjunto de objetivos de melhorias, obtidos no estágio "Definir objetivos de implantação de melhorias", o planejamento necessário referente ao atendimento desses resultados é realizado. $\mathrm{O}$ principal artefato utilizado neste estágio é a lista priorizada de objetivos de melhoria, conforme exemplo apresentado na Figura 6. Por meio dela é possível estabelecer o planejamento da iteração, indicando objetivos factíveis de serem implantados em um determinado período de tempo.

\begin{tabular}{c|l}
\hline Prioridade & Objetivos de Melhoria \\
\hline 1 & Minimizar distrações no ambiente de trabalho. \\
2 & Documentar os objetivos de desempenho para o trabalho estabelecido. \\
3 & Estabelecer mecanismos para gerenciar problemas de desempenho. \\
4 & Premiar ou reconhecer o desempenho excepcional. \\
5 & Realizar a transição de papéis, funções e equipes de maneira planejada e organizada. \\
\hline
\end{tabular}

Figura 6 - Exemplo de lista de melhorias priorizadas.

\subsection{Estágio: Executar implantação de melhorias}

O estágio "Executar implantação de melhorias" se preocupa com a promoção da implantação de melhoria propriamente dita e com o acompanhamento dessa implantação. As informações relevantes obtidas, tanto em relação a sucesso quanto a insucessos, são registradas. Diversos artefatos são gerados durante a execução deste estágio buscando evidenciar a execução das práticas esperadas pelos objetivos estabelecidos no estágio anterior ("Planejar iteração de implantação de melhorias"). Na Figura 7 é apresentado um trecho de uma planilha gerada para atender práticas da área de processo "Treinamento e Desenvolvimento" do nível 2 do modelo P-CMM. Nesse caso, também neste estágio são produzidos o material necessário para a realização do treinamento bem como o treinamento propriamente dito. 


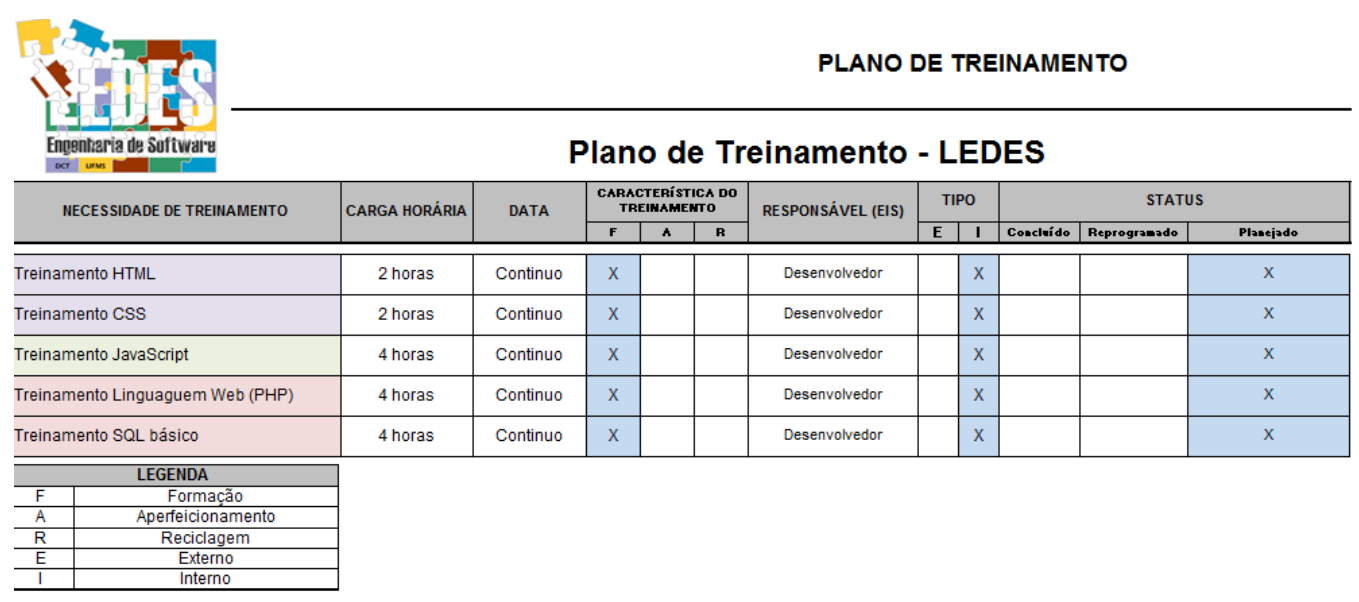

Figura 7 - Trecho do plano de treinamento

\section{Estudo de Caso}

Para analisar o uso do arcabouço Aim-P quanto ao apoio na melhoria de pessoas em uma organização real, um estudo de caso está sendo conduzido no Laboratório de Engenharia de Software (LEDES) da Faculdade do Computação (Facom-UFMS). O LEDES é um laboratório de pesquisa na área de Engenharia de Software, constituindose de um ambiente para a aplicação prática de conceitos fundamentais de desenvolvimento de software. Atualmente o LEDES conta com aproximadamente 30 colaboradores (sendo cerca de dezesseis graduandos, oito graduados e quatro docentes), quatro projetos de software e cinco projetos de pesquisa.

O estudo de caso está sendo realizado desde agosto de 2013 com a equipe de desenvolvimento do LEDES que é composta por pessoas de diversos níveis acadêmicos, desde doutores até recém-ingressos em cursos de Computação. Os membros são divididos hierarquicamente em papéis de acordo com suas experiências, habilidades e conhecimentos: a) desenvolvedor iniciante: papel atribuído a pessoas que entraram recentemente nos projetos, sendo geralmente graduandos com pouca afinidade com as tecnologias de desenvolvimento adotadas e que não possuem familiaridade com os sistemas de software do projeto em que atua; b) desenvolvedor avançado: papel desempenhado por pessoas que alcançaram um bom nível de autonomia de desenvolvimento, tanto em relação às tecnologias quanto em relação ao conhecimento do sistema de software e das regras de negócio do domínio do mesmo; c) líderes de equipe: papel assumido por pessoas que detém um grau de conhecimento superior aos outros desenvolvedores, devendo possuir maturidade, capacidade de liderança e conhecimento suficiente para gerir os demais desenvolvedores; e d) gestores: papel atribuído a pessoas com alto grau acadêmico e com profundo conhecimento em Engenharia de Software.

Atualmente a equipe é formada por três gestores, quatro líderes (cada um responsável por equipes que variam de dois a cinco desenvolvedores), oito desenvolvedores iniciantes (desses, três deles estão distantes geograficamente dos demais) e cinco desenvolvedores avançados.

Para definir o estudo de caso, foi utilizado o método GQM (Goal, Question e Metric) (BASILI, 1994), uma abordagem orientada a metas e utilizada em Engenharia de Software para apoiar a medição de produtos e processos de software. 
O objeto do estudo de caso é o arcabouço Aim-P e o objetivo é avaliar a aplicação desse arcabouço no âmbito de uma pequena organização de desenvolvimento de software. $\mathrm{O}$ estudo de caso tem como foco qualitativo avaliar a capacidade do Aim$\mathrm{P}$ na melhoria de pessoas em relação aos objetivos e práticas esperadas das áreas de processos do nível 2 do $\mathrm{P}-\mathrm{CMM}^{3}$. O estudo tem como perspectiva apoiar a melhoria de pessoas no LEDES, mais especificamente os desenvolvedores locais do projeto SIGFAP (Sistema de Informação de Gestão de Fundações de Amparo à Pesquisa) (TURINE et al., 2011). Como contexto, o estudo de caso está sendo conduzido pelo autor deste trabalho, tendo a colaboração de um docente como consultor e de três líderes de desenvolvimento da organização. Esses líderes, juntamente com o autor deste trabalho, fazem parte do grupo de implantação de melhorias.

Os participantes do estudo de caso são divididos em dois tipos: o primeiro engloba o grupo de implantação de melhorias de pessoas e o segundo refere-se aos desenvolvedores que participaram dessa implantação. A seleção do projeto e dos membros participantes do estudo de caso foi realizada levando em consideração o projeto do LEDES com maior número de pessoas envolvidas. Tendo como base essa premissa, o projeto SIGFAP foi selecionado. Para compor o grupo de implantação foram selecionados os três líderes de equipes locais e os respectivos membros dessas equipes para atuarem como participantes da implantação de melhoria de pessoas, perfazendo um total de 16 pessoas. Salienta-se que todos os líderes são pós-graduados em Ciência da Computação na área de Engenharia de Software.

Antes do início do estudo de caso foi ministrado um treinamento visando capacitar a equipe de implantação para o uso adequado do arcabouço Aim-P. Para isso, foi dado um treinamento de três horas sobre o Aim-P e sobre o modelo de melhoria de pessoas P-CMM, focando os objetivos da área de processo Alocação de Colaboradores (Staffing) do nível 2. Não houve a necessidade de um treinamento muito longo, pois todos os membros da equipe de implantação conheciam pelo menos um modelo de maturidade de processo de desenvolvimento de software existente.

Além disso, na fase de planejamento, foram observadas as possíveis ameaças à validade do estudo de caso. A maior ameaça encontrada no planejamento foi a participação do condutor do estudo de caso na organização, uma vez que ele também é líder de equipe no LEDES. Para evitar problemas na validade do estudo de caso ele evitou participações desnecessárias, atuando somente como um consultor no uso do arcabouço e também em algumas tomadas de decisões estratégicas da organização.

Considerando a aplicação do GQM, a primeira iteração do estudo de caso buscou responder três questões: (1) As tarefas do arcabouço Aim-P previstas para serem realizadas no projeto de implantação de melhorias foram realizadas? (2) Os artefatos das tarefas previstas foram gerados? (3) Os objetivos de melhorias previstos foram alcançados?

Para fins da execução do arcabouço Aim-P, de acordo com a realidade do LEDES e em especial dos membros do projeto SIGFAP, os seguintes estágios do Aim-P estão sendo avaliados pelo estudo de caso: "Definir Objetivos de Implantação de

\footnotetext{
${ }^{3}$ As práticas utilizadas no estudo de caso foram selecionadas durante a fase de definição de objetivos de implantação de melhorias de pessoas do arcabouço Aim-P, que foi a primeira a ser executada e que colaborou para a definição do foco qualitativo do estudo de caso conduzido.
} 
Melhorias"; "Planejar iteração de implantação de melhorias"; "Executar implantação de melhorias"; e estágio "Avaliar pessoas". Alguns dos artefatos elaborados durante a execução deste estudo de caso estão apresentados na Seção 4.

Para averiguar se o objetivo da primeira iteração do estudo de caso foi alcançado, cada uma das questões estabelecidas foi respondida, tomando como base as informações obtidas a partir da execução do estudo de caso realizado.

Assim, com a execução do estudo de caso observou-se que todas as atividades previstas para a primeira iteração do projeto de melhorias de pessoas do LEDES foram executadas. O estágio "Estabelecimento de Equipe" estava fora do escopo do estudo de caso, pois as equipes já haviam sido estabelecidas. Conforme previsto, os artefatos das atividades previstas foram gerados e utilizados. Os modelos oferecidos pelo arcabouço foram utilizados para gerar os formulários de avaliação, o relatório de execução da avaliação, o questionário para identificar necessidades de melhorias e relatório estatístico das necessidades de melhorias. Para atender todos os objetivos estabelecidos inicialmente no planejamento do projeto de implantação de melhoria, era necessário atender nove práticas esperadas, das quais quatro referem-se a área de processo Alocação de Colaboradores (Staffing) e cinco estão relacionadas a área de processo Treinamento e Desenvolvimento. Na primeira avaliação do estudo de caso, seis práticas foram atendidas totalmente e uma foi atendida parcialmente, representando cerca de $70 \%$ das práticas previstas. Duas práticas esperadas não foram atendidas, pois é necessário que ocorram algumas execuções do que foi planejado para que se possa acompanhar o andamento em relação ao planejado. Dessa forma, a partir dos resultados preliminares obtidos observou-se que o arcabouço Aim-P provê apoio na melhoria de pessoas.

\section{Conclusões}

A literatura apresenta lacunas a respeito do tema abordado por este trabalho, não somente em relação a mecanismos de apoio a implantação de melhoria de gestão de pessoas, como também em relação a modelos de maturidade para esse fim. Observa-se que as pesquisas no contexto de modelos de maturidade de processos de software estão bem mais avançadas, isso pode ser confirmado pela existência de diversos modelos renomados e bastante utilizados, como é o caso do CMMI e do MPS.BR.

Espera-se que com o apoio do arcabouço Aim-P, proposto neste trabalho, a implantação de melhorias de pessoas seja divulgada e praticada nas organizações de software brasileiras de forma que elas possam melhorar a gestão de suas pessoas despendendo a menor quantidade possível de recursos. A área de pesquisa de melhoria de pessoas e de equipes de desenvolvimento de software é pouco explorada, apesar de não ser muito recente. Por isso, espera-se que a partir deste trabalho outros sejam desenvolvidos de maneira a contribuir para o avanço da área.

Durante a condução do estudo de caso, observou-se a necessidade da criação de um repositório de modelos que possa ser utilizado como entrada para os estágios do arcabouço ou como apoio para ajudar a evidenciar um objetivo esperado de uma determinada prática. Observou-se também a importância de conduzir mais avaliações do Aim-P em outras organizações para comprovar estatisticamente a eficiência do mesmo.

Os seguintes trabalhos futuros são sugeridos para dar prosseguimento a este trabalho: i) desenvolver um ambiente, composto por um conjunto de ferramentas, que 
apoie a condução do projeto de implantação de melhorias de pessoas e estabelecimento de equipes proposto por este trabalho; ii) evoluir e propor novos modelos de artefatos; iii) especificar e desenvolver um repositório de estratégias de implantação de melhoria de pessoas com as respectivas lições aprendidas durante implantações anteriores; e iv) conduzir estudos empíricos em diversas organizações para produzir resultados com significância estatística.

\section{Referências}

AGILE ALLIANCE. The Agile Manifesto. Manifesto for Agile Software Development, 2001. Disponível em: <www.agilemanifesto.org>. Acesso em: fevereiro 2014.

BASILI, V., CALDIERA, G., and ROMBACH, D. Experience Factory. Encyclopedia of Software Engineering Volume 1:469-476, Marciniak, J. ed. John Wiley \& Sons, 1994.

BECKER, B. E.; HUSELID, M. A.; ULRICH, D. The HR Scorecard: Linking People, Strategy, and Performance. Boston: Harvard Business School Press, 2001.

BENITES, M. IAMPS: Um processo de apoio ao uso de métodos ágeis em conjunto com o MPS-BR. Dissertação de Mestrado, Facom, UFMS, 2012.

BENITES, M.; OLIVEIRA, L.; PAIVA, D.; CAMARGO, D.; GOMES, P.; CAGNIN M. I. Implantação de Resultados Esperados do Processo Gerência de Projetos com o apoio do Scrum no Setor Público In: V Workshop de Gerenciamento de Projetos de Software (WGPS) em conjunto com o Simpósio Brasileiro de Qualidade de Software (SBQS), Fortaleza-CE, 2012.

CHEN, Y.; HSIEH, K.; WU, M. The Study of Second Level of People Capability Maturity Model on the Industrial Control Industry in Taiwan. Journal of Quality, v. 19, n. 1, p. 1, 2012.

CURTIS, W.; HEFLEY, W.; MILLER, S. People CMM (Version 2) (CMU/SEI-2009TR-003). Software Engineering Institute, Carnegie Mellon University, 2009.

DE GEUS, A. The Living Company. Boston: Harvard Business School Press, 2002.

GALVÃO, M. R.; MAGAlHÃES, A. L. C. C. Gestão de Competências: a Caminho da Maturidade. In: VII Simpósio Internacional de Melhoria de Processos de Software, 2005.

HEFLEY, W. E.; CURTIS, B.; MILLER, S.; KONRAD, M. People Capability Maturity Model (P-CMM): Incorporating Human Resources into Process Improvement Programs. In: Annual international symposium of the National Council on Systems Engineering. National Council on Systems Engineering, 1995. p. 559-566.

HUMPHREY, WATTS. Team Software Process (TSP). CMU/SEI-2000-TR-023. SEI, Carnegie Mellon University, 2000.

ISO/IEC 15504. ISO/IEC 15504: Part 3 and ISO/IEC 15504: Part 4, Geneve: ISO, 2004.

MCFEELEY, B. IDEAL: A User's Guide for Software Process Improvement. Technical Report CMU/SEI-96-HB-001, SEI - Carnegie Mellon University, 1996.

OLIVEIRA, L. M. Aim-P: Um Arcabouço para Apoiar a Implantação de Melhoria de Pessoas. Dissertação de mestrado. Faculdade de Computação-UFMS, Campo 
Grande-MS, 2013. Disponível em: http://facom.ufms.br/gestor/titan.php?target $=$ openFile \&fileId $=1365$

SANTOS, G.; MONTONI, M.; FIGUEIREDO, S.; ROCHA, A. R. (2007). SPI-KM Lessons Learned from Applying a Software Process Improvement Strategy Supported by Knowledge Management. In: 8th International Conference on Product Focused Software Process Improvement, p. 81-95, 2007.

SCAMPI. Standard CMMI Appraisal Method for Process Improvement (SCAMPI). Version 1.3: Method Definition Document (CMU/SEI-2011-HB-001). SEI, Carnegie Mellon University, 2011.

SEI - SOFTWARE ENGINEERING INSTITUTE. CMMI for Development (CMMIDEV), Version 1.3, Technical Report CMU/SEI-2010-TR-033. Pittsburgh, PA: SEI, Carnegie Mellon University, 2010.

SOFTEX - ASSOCIAÇÃO PARA PROMOÇÃO DA EXCELÊNCIA DO SOFTWARE BRASILEIRO - SOFTEX. MPS.BR - Guia de Avaliação MA-MPS, Setembro 2013.

SOFTEX - ASSOCIAÇÃO PARA PROMOÇÃO DA EXCELÊNCIA DO SOFTWARE BRASILEIRO. MPS.BR Guia Geral: 2012, Agosto 2012.

SOMMERVILLE, I. Engenharia de Software. 7ª ed. São Paulo: Addison Wesley, 2010.

TUCKMAN, B. Developmental sequence in small groups. Psychological Bulletin 63, 1965. Reprinted with permission in Group Facilitation, Spring 2001. 